\title{
„Hauptsache mir wird geholfen“: Patientenperspektive und randomisierte Studien
}

\author{
"I Need a Doctor who Cures my Symptoms": Patients Perspectives and Randomised Trials
}

Randomisierte, kontrollierte Studien (RCT) sind der Gold Standard zur Beurteilung der Wirksamkeit von Therapien. Leider sind RCTs aufwändig und teuer und werden im Rahmen größerer Forschungsförderungsprogramme nur zu als besonders wichtig angesehenen Fragestellungen realisiert $[2,7]$. Zu vielen klinisch relevanten Fragestellungen fehlen RCTs obwohl die Notwendigkeit erkannt ist $[1,8]$. Bauchschmerzen gehören zu den häufigsten Beschwerden im Kindesalter. Sie können spezifische Ursachen haben, die einer gezielten Behandlung bedürfen [3,5]. Eine besondere Herausforderung ist aber die Behandlung idiopathischer Bauchschmerzen. Es ist ein besonderer Verdienst von Wirth et al. eine randomisierte Studie zur Therapie von idiopathischen Bauchschmerzen durch eine Fruktose reduzierte Diät konzipiert und durchgeführt zu haben [9]. Diese Studie fokussiert patientenrelevante Endpunkte: Häufigkeit und Schwere der Beschwerden.

Die Ergebnisse sind eindrucksvoll - die Häufigkeit und Schwere der Symptome werden fast halbiert. Die Messung erfolgt, wie bei diätetischen Interventionen nur schwer anders möglich, ohne Verblindung bzw. ohne Plazebo auf einer subjektiven Skala. Und genau das könnte eine Limitation der Studie sein. Wie beim systematischen Vergleich von verschiedenen 3-armigen Studien mit Verum, Plazebo und ohne Intervention gezeigt wurde [4], sind die Ergebnisse von Studien ohne Plazebo immer dann besonders gefährdet, Plazeboeffekte zu berichten, wenn die Endpunkte subjektive Beschwerden waren, die auf Skalen erfasst wurden. Genau dies ist bei der Studie von Wirth et al. der Fall. Profitierten die Patienten allein vom Plazeboeffekt oder sind Plazeboeffekte die wesentliche Therapiekomponente? Als Wissenschaftler würden wir es schon gerne genauer wissen - aus Patientensicht scheint es jedoch primär völlig gleich, ob die Effekte der Wirksubstanz oder der „Verpackung der Wirksubstanz“ zuzuschreiben sind.

Viele der Interventionen im Kindesalter, wie Krankengymnastik, Verhaltenstraining, Ergotherapie, Diäten sind nicht medikamentös und können kaum in Plazebo kontrollierten Studien mit Verblindung geprüft werden. In einer kürzlich veröffentlichten Metaanalyse konnten wurde gezeigt, dass z. B. spezifische Trainingsprogramme zur Verbesserung kognitiver Leistungen immer nur dann effektiv waren, wenn in der Kontrollgruppe keine Therapie erfolgte. Erhielt die Kontrollgruppe eine
Plazebotherapie waren keine Effekte mehr erkennbar [6]. Sind deshalb diese Trainingsprogramme deshalb obsolet? Ohne Zweifel sind sie wirksam - auch wenn Zuwendung anderer Art wahrscheinlich genau so effektiv ist.

Der Beitrag von Wirth et al. liefert noch eine weitere wichtige Erkenntnis: Die Effekte der Fruktose reduzierten Diät waren völlig unabhängig davon, ob eine Fruktose Malabsorption vorliegt oder nicht. Eine wesentliche Stärke der Arbeit: dies wurde erst dann untersucht, als die klinische Beurteilung der Intervention schon abgeschlossen war: Verzerrung der Ergebnisse durch Erwartungshaltung bei bekannter Fruktose Malabsorption wurde so ausgeschlossen. So führt die Arbeit das Paradigma „Kein Versuch mit einer Fruktose armen Diät ohne vorherigen Nachweis einer Fruktose Malabsorption“ ad absurdum: Der Effekt der Intervention ist völlig unabhängig vom Ergebnis des diagnostischen Standards für Fruktose Malabsorption.

Fazit: Bei Kindern mit idiopathischen Bauchschmerzen ist ein Therapieversuch mit Fruktose reduzierter Diät gerechtfertigt - auf einen Fruktose Belastungstest kann sicher verzichtet werden: „Hauptsache mir wird geholfen“. Es gibt nun eine Menge von Interventionen im Kindesalter, bei denen eine Sicherung der Therapieeffekte durch randomisierte, Plazebo kontrollierte Studien nicht möglich ist. Bei Interventionen ohne potentielle Nebenwirkungen kann man damit leben - auch aus Patientensicht. Risiken einer zeitlich befristeten Fruktose armen Diät scheinen gering zu sein. Wenn nun aber hieraus das Diagnose Stigma „Fruktose Malabsorption“ wird und deshalb langfristig Obst und Gemüse gemieden werden, sind Nebenwirkungen jedoch keinesfalls auszuschließen.

Nur in einer 3-armigen Studie zur Fruktose reduzierten Diät bei idiopathischen Bauchschmerzen mit Fruktose reduzierter Diät, Nonsense Diät und Beobachtung ohne Diät können Plazeboeffekte sicher erkannt werden. Die wissenschaftliche Neugier und der Enthusiasmus von Kollegen wie Stefan Wirth braucht ein Programm zur Finanzierung solcher Studien. Eine Ausschreibung für kontrollierte Studien zur Evaluation von Interventionen auch für nicht „vitale“ Indikationen im Kindesalter ist überfällig. 


\section{Literatur}

1 Anders EF, Findeisen A, Lode HN et al. Acupuncture for treatment of acute vomiting in children with gastroenteritis and pneumonia. Klin Padiatr 2012; 224: 72-75

2 Bochennek K, Dantonello T, Koscielniak E et al. Response of children with stage IV soft tissue sarcoma to topotecan and carboplatin: a phase II window trial of the cooperative soft tissue sarcoma group. Klin Padiatr 2013; 225: 309-314

3 Flemming GM, Toth G, Gebauer C et al. Crohns' disease in a patient with juvenile idiopathic arthritis after starting etanercept therapy - causal link or only temporal coincidence? Klin Padiatr 2013; 225: 350-351

4 Hrobjartsson A, Gotzsche PC. Is the placebo powerless? An analysis of clinical trials comparing placebo with no treatment. N Engl J Med 2001; 344: 1594-1602

5 Jeske M, Lohse P, Kallinich T et al. Genotype-phenotype and genotypeorigin correlations in children with mediterranean fever in Germany - an AID-net study. Klin Padiatr 2013; 225: 325-330
6 Karch D, Albers L, Renner G et al. The Efficacy of Cognitive Training Programs in Children and Adolescents A Meta-analysis. Dtsch Arztebl Int 2013; 110: U623-U643

7 Schrappe M, Moricke A, Reiter A et al. Key treatment questions in childhood acute lymphoblastic leukemia: results in 5 consecutive trials performed by the ALL-BFM study group from 1981 to 2000. Klin Padiatr 2013; 225 (Suppl 1): S62-S72

8 Tibussek D, Distelmaier F, von Kries $R$ et al. Pseudotumor cerebri in childhood and adolescence - results of a Germany-wide ESPED-survey. Klin Padiatr 2013; 225: 81-85

9 Wirth S, Klodt Ch, Wintermeyer Ph et al. Positive or negative fructose breath test results do not predict response to fructose restricted diet in children with recurrent abdominal pain: Results from a prospective randomized trial. Klin Padiatr 2014; 226: 268-273 